\title{
Common Structural Polymorphisms in Human Erythrocyte Spectrin
}

\author{
W. J. Knowles, M. L. Bologna, J. A. Chasis, \\ S. L. Marchesi, and V. T. Marchesi \\ Departments of Pathology and Internal Medicine, \\ Yale University, New Haven, Connecticut 06510
}

A

bstract. Restricted tryptic digestion of erythrocyte spectrin at $4^{\circ} \mathrm{C}$ followed by two-dimensional (isoelectric-focusing/sodium dodecyl sulfate) polyacrylamide electrophoresis yields highly reproducible maps of approximately 50 peptides with molecular weights between 80,000 and 12,000 . Based on molecular weight and isoelectric point (pI), each unique $\alpha$ - and $\beta$-subunit domain can be identified and compared with spectrin peptides from other individuals. The $\alpha$-subunit of spectrin from 60 Caucasian donors contains a 46,000-mol-wt tryptic domain, called $\alpha$ II-T46, Type 1; more extensive tryptic digestion of this domain generates peptides with molecular weights of $35,000,30,000,25,000$, and 16,000 . Spectrin from 29 of 37 black donors representing 14 kindreds shows variation in the molecular weight and/or $\mathrm{p} I$ of peptides from the $\alpha$ II domain. In the most common form, Type 2, $\alpha$ II tryptic peptides are increased in molecular weight by 4,000 , and the $\mathrm{p} I$ becomes more basic. Other $\alpha$ II variants are characterized by either the 4,000 increase in molecular weight (Type 3) or by the basic shift in $\mathrm{p} I$ (Type 4). When limit peptide maps of intermediate-sized tryptic and $\mathrm{CNBr}$ peptides from the $\alpha \mathrm{II}-$ domain Types 1 and 2 are compared, a consistent alteration in the chromatographic mobility of one limit peptide is observed.

Polymorphism in the $\alpha \mathrm{II}$ subunit of spectrin did not itself produce anemia, nor did it appear to alter the expression of an underlying hereditary spherocytosis or elliptocytosis. In six family studies, the $\alpha$ II 46,000 mol-wt variations observed were consistent with Mendelian inheritance.

\section{Introduction}

It is generally agreed that spectrin is essential in determining the properties of the red cell membrane, including its shape and

Received for publication 7 July 1983 and in revised form 4 November 1983.

J. Clin. Invest.

(c) The American Society for Clinical Investigation, Inc. 0021-9738/84/04/0973/07 \$1.00

Volume 73, April 1984, 973-979 deformability. Structural analysis of the spectrin molecule has been difficult because of the large size of its two nonidentical subunits, $\alpha(240,000 \mathrm{~mol} \mathrm{wt})$ and $\beta(225,000 \mathrm{~mol} \mathrm{wt})$. In such a large and complex molecule, small changes in conformation or physicochemical behavior would not necessarily cause significant alterations in the properties of the native molecule. Our laboratory has recently developed a technique for analyzirg spectrin structure; this technique uses limited tryptic digestion of spectrin at $0^{\circ} \mathrm{C}$, followed by two-dimensional electrophoresis (isoelectric-focusing, sodium dodecyl sulfate-polyacrylamide gel electrophoresis [IEF/SDS-PAGE]). ${ }^{1}$ This procedure results in remarkably reproducible maps of approximately 50 intermediate-sized peptides whose molecular weights range from 80,000 to 12,000 . These peptides may be enzymatically digested or chemically cleaved and the resultant limit peptide analyzed by high-resolution mapping techniques. With these procedures, it is possible to assign the origins of all peptides to the $\alpha$ - or $\beta$ subunit, to determine relationships between peptides at various stages of digestion, and to arrange the unique peptides linearly, producing a domain structure for each spectrin subunit (1).

These techniques have been applied to the study of red cell membranes of patients with hereditary spherocytosis (HS) and hereditary elliptocytosis (HE) and their variants. We and others $(2,3)$ have recently demonstrated that patients with severe poikilocytic hemolytic anemia (hereditary pyropoikilocytosis, HPP) have abnormalities in the 80,000-mol-wt domain of the $\alpha$-chain.

In this study, we describe the occurrence of polymorphisms in the $\alpha$ II domain that involve changes in both the molecular weight and the isoelectric point $(\mathrm{p} I)$. These polymorphisms have been found only in the black population, are relatively common, and appear not to be correlated with any clinical abnormality.

The first family in which we demonstrated an $\alpha$ II polymorphism was a black family with classical HS. Heterozygous and homozygous forms of the $\alpha$ II variant were detected and they occurred in predictable Mendelian fashion, but segregated independently of spherocytosis. We subsequently studied 37 black individuals from 14 kindreds and we discovered a polymorphic $\alpha$ II domain in 29 of these individuals, the variations being in molecular weight or $\mathrm{p} I$ or both, seen in single or double

1. Abbreviations used in this paper: DFP, diisopropyl fluorophosphate; HE, hereditary elliptocytosis; HPP, hereditary pyropoikilocytosis; HS, hereditary spherocytosis; IEF/SDS-PAGE, isoelectric-focusing, SDSpolyacrylamide gel electrophoresis. 


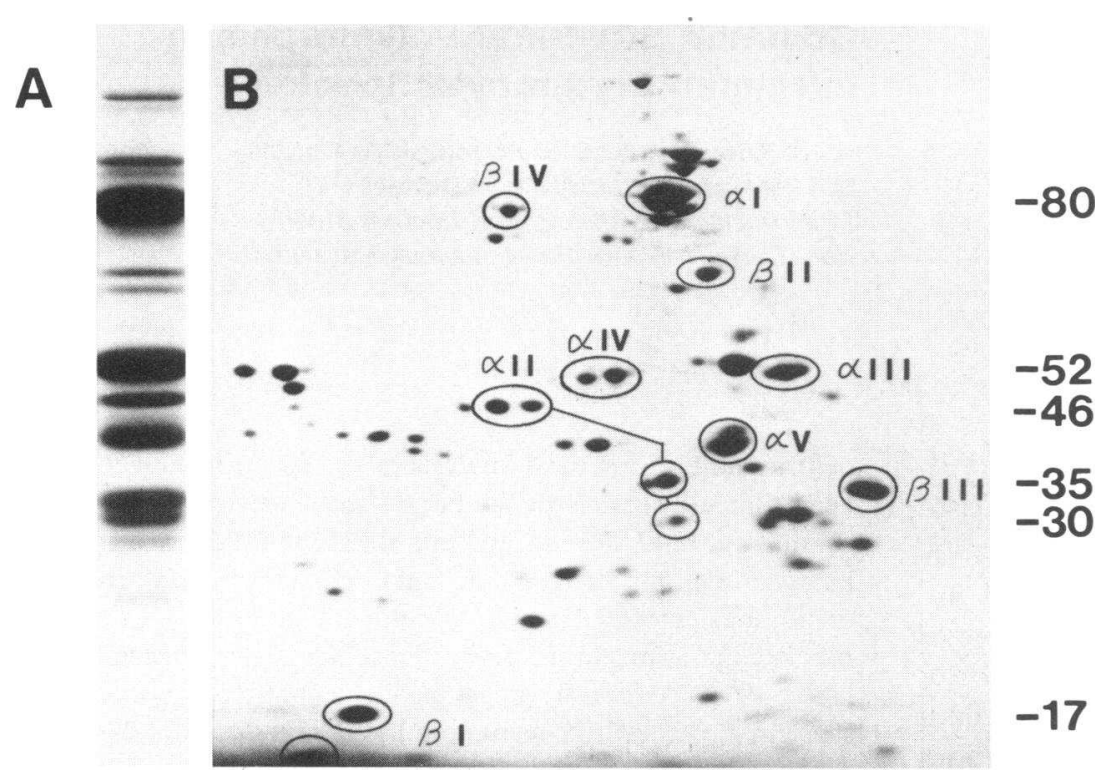

Figure 1. Electrophoresis of spectrin peptide generated by limited tryptic digestion at $4^{\circ} \mathrm{C} .(A) 75$ $\mu \mathrm{g}$ lyophilized tryptic digest in $2 \%$ SDS, $2 \% \beta$ mercaptoethanol electrophoresed on a $10-15 \%$ acrylamide gradient according to Laemmli (5). (B) $200 \mu \mathrm{g}$ tryptic digest electrophoresed in two dimensions (IEF/SDS) according to O'Farrell (6). The $\mathrm{pH}$ gradient (horizontal) extends from 7.2 to 4.5 (left to right); the acrylamide gradient (vertical) is from 10 to $15 \%$. Unique domains in the $\alpha$ and $\beta$-chains are circled. Lower molecular weight tryptic digestion products of the $\alpha$ II-T46 domain are connected by lines from the parent domain. Numbers indicate molecular weight in thousands. heterozygote form. We made no attempt to determine the actual frequency of these variants in the black population.

\section{Methods}

\section{Spectrin preparation}

Blood was drawn into acid citrate dextrose anticoagulant and stored at $4^{\circ} \mathrm{C}$ until processed, no more than $24 \mathrm{~h}$. Erythrocyte membranes were prepared and spectrin was extracted as previously described (4).

\section{Tryptic digestion of spectrin}

$200-\mu \mathrm{g}$ aliquots of spectrin in $200 \mu \mathrm{l}$ were dialyzed against $20 \mathrm{mM}$ Tris$\mathrm{HCl}, \mathrm{pH} 8.0,1 \mathrm{mM}$ 2-mercaptoethanol for $20 \mathrm{~h}$ at $4^{\circ} \mathrm{C}$. Trypsin $(N$ tosyl-L-phenylalanine chloromethyl ketone-treated, $206 \mu \mathrm{m} / \mathrm{mg}$; Worthington Biochemical Corp., Freehold NJ) was added at an enzyme/ substrate ratio of $1: 20(\mathrm{wt} / \mathrm{wt})$. The reaction was carried out at $4^{\circ} \mathrm{C}$ for

A

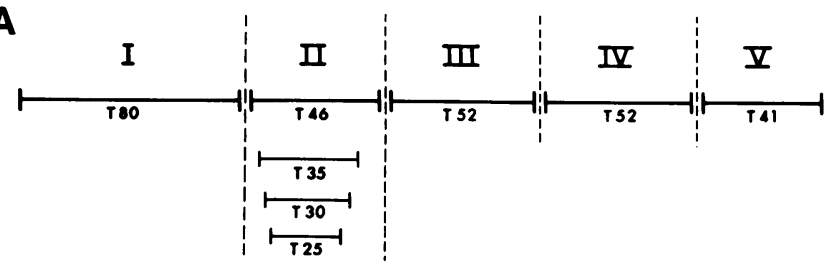

B

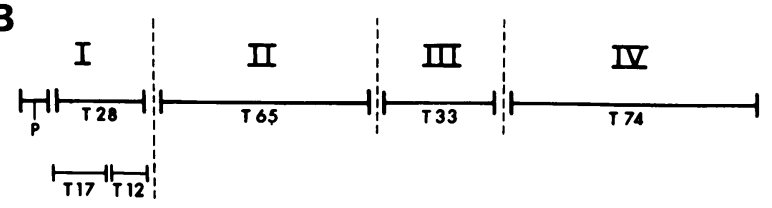

Figure 2. Diagrammatic representation of the $\alpha$ - and $\beta$-chains of spectrin deduced from chemical and enzymatic cleavage and mapping of overlap peptides (8-10). Numbers indicate molecular weight in thousands.
90 min and terminated by adding diisopropyl fluorophosphate to a final concentration of $1 \mathrm{mM}$ and cooling to $-80^{\circ} \mathrm{C}$. The tryptic digests were lyophilized and stored at $-20^{\circ} \mathrm{C}$ for further use.

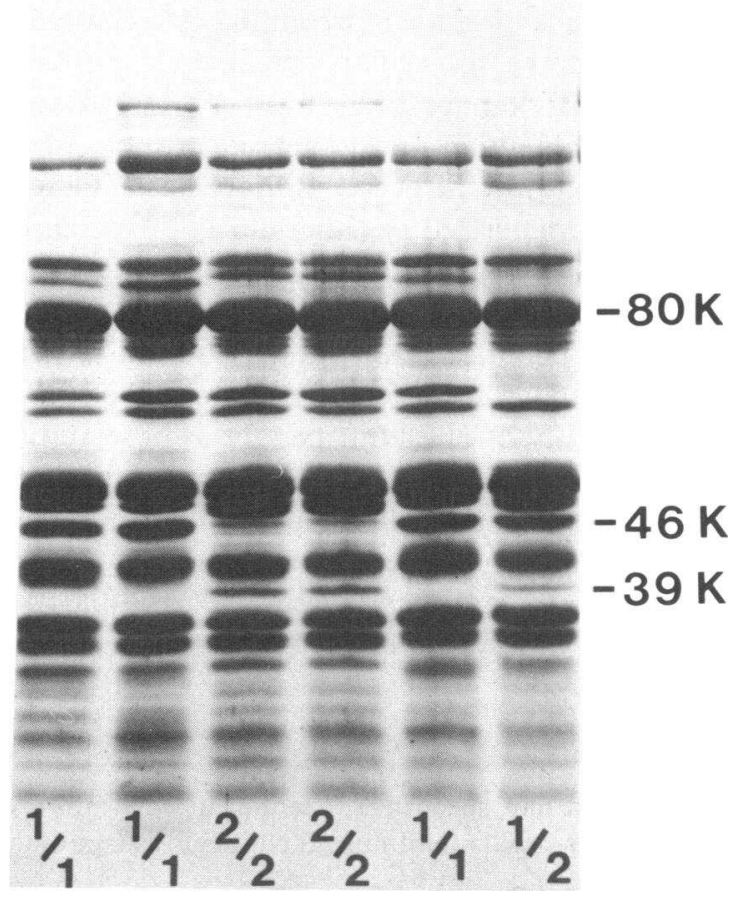

Figure 3. Type 2 variation in the $\alpha$ II-T46 domain of spectrin. Intermediate tryptic peptides of spectrin $(75 \mu \mathrm{g})$ from members of a family with $\alpha$ II Type 2 polymorphism are electrophoresed on a $10-15 \%$ acrylamide gradient by SDS-PAGE. 1/1: spectrin homozygous for $\alpha \mathrm{II}$ Type 1. 2/2: spectrin homozygous for $\alpha$ II Type 2. 1/2: spectrin doubly heterozygous for $\alpha$ II Types 1 and $2.39 \mathrm{~K}, 39,000$-mol-wt peptide; $46 \mathrm{~K}, 46,000$-mol-wt peptide; $80 \mathrm{~K}, 80,000$-mol-wt peptide. 


\section{Polyacrylamide gel electrophoresis}

One-dimensional electrophoresis. $75 \mu \mathrm{g}$ of spectrin tryptic peptides prepared as described above was solubilized in $2 \%$ SDS, $2 \% 2$-mercaptoethanol and electrophoresed according to Laemmli (5) on acrylamide gradients, as specified in Figure legends.

Two-dimensional electrophoresis (IEF/SDS-PAGE). $200 \mu \mathrm{g}$ of lyophilized spectrin tryptic digests was solubilized in $75 \mu 19 \mathrm{M}$ urea, $2 \%$ Triton X-100, 5\% 2-mercaptoethanol, and 2.4\% ampholines, and focused on $3 \times 120 \mathrm{~mm}$ polyacrylamide gels according to O'Farrell (6). The $\mathrm{pH}$ gradient extended from 7.2 to 4.5 and was formed by mixing equal volumes of ampholytes (LKB Instruments, Inc., Rockville MD) at $\mathrm{pH}$ 4-6, pH 5-7, and $\mathrm{pH} 3.5-10$ (2.4\% final ampholyte concentration). After electrophoresis for $5,700 \mathrm{~V} / \mathrm{h}$, the gels were removed and equilibrated for $10 \mathrm{~min}$ in $10 \%$ glycerol, 3\% SDS, $1 \mathrm{mM}$ EDTA, and 2\% 2mercaptoethanol before electrophoresis in the second dimension on a $10-15 \%$ acrylamide gradient using Laemmli buffers (5). Molecular weights were determined by reference to known protein standards.

\section{CNBr cleavage}

Lyophilized spectrin $(1 \mathrm{mg}$ ) was dissolved in $70 \%$ formic acid. A 500 fold molar excess of CNBr (Pierce Chemical Co., Rockford, IL) with respect to methionine was added and the reaction was allowed to proceed overnight at $37^{\circ} \mathrm{C}$. The reaction was terminated by drying with $\mathrm{N}_{2}$, followed by repeated resuspension in water and lyophilization. The samples were then subjected to two-dimensional electrophoresis according to O'Farrell (6) as described above.

\section{${ }^{125}$ I-peptide mapping}

Two-dimensional peptide maps were prepared by the method of Elder et al. (7) using Coomassie Blue-stained proteins cut from acrylamide gels and labeled with $\mathrm{Na}^{125}$ (New England Nuclear, Boston, MA) as previously described $(8,9)$. Iodinated gel slices were digested with 50 $\mu \mathrm{g}$ of $\alpha$-chymotrypsin (Worthington Biochemical Corp.) in $1 \mathrm{ml}$ of 50 $\mathrm{mM}$ ammonium bicarbonate, $\mathrm{pH} 8.0$, for $24 \mathrm{~h}$ at $37^{\circ} \mathrm{C}$. The lyophilized samples were subjected to two-dimensional peptide mapping; details of this procedure have been described earlier $(8,9)$.

\section{Results}

Two-dimensional electrophoresis of intermediate-sized tryptic peptides of spectrin. We have isolated spectrin from the red cells of 97 individuals and from 10 cord blood samples, cleaved it by limited tryptic digestion at $0^{\circ} \mathrm{C}$, and analyzed the resultant intermediate-sized peptides by one- and two-dimensional electrophoresis. The peptide patterns obtained are highly reproducible, and blood given by a single donor on multiple occasions has always produced the same spectrin peptide patterns, whether normal or abnormal. A typical spectrin digest can be seen on one-dimensional SDS-PAGE in Fig. $1 A$ and on two-dimensional IEF/SDS-PAGE in Fig. $1 B$. It is apparent from Fig. 1 that the complexity of the spectrin digest requires two-dimensional electrophoresis to resolve all spectrin domains and subdomains,
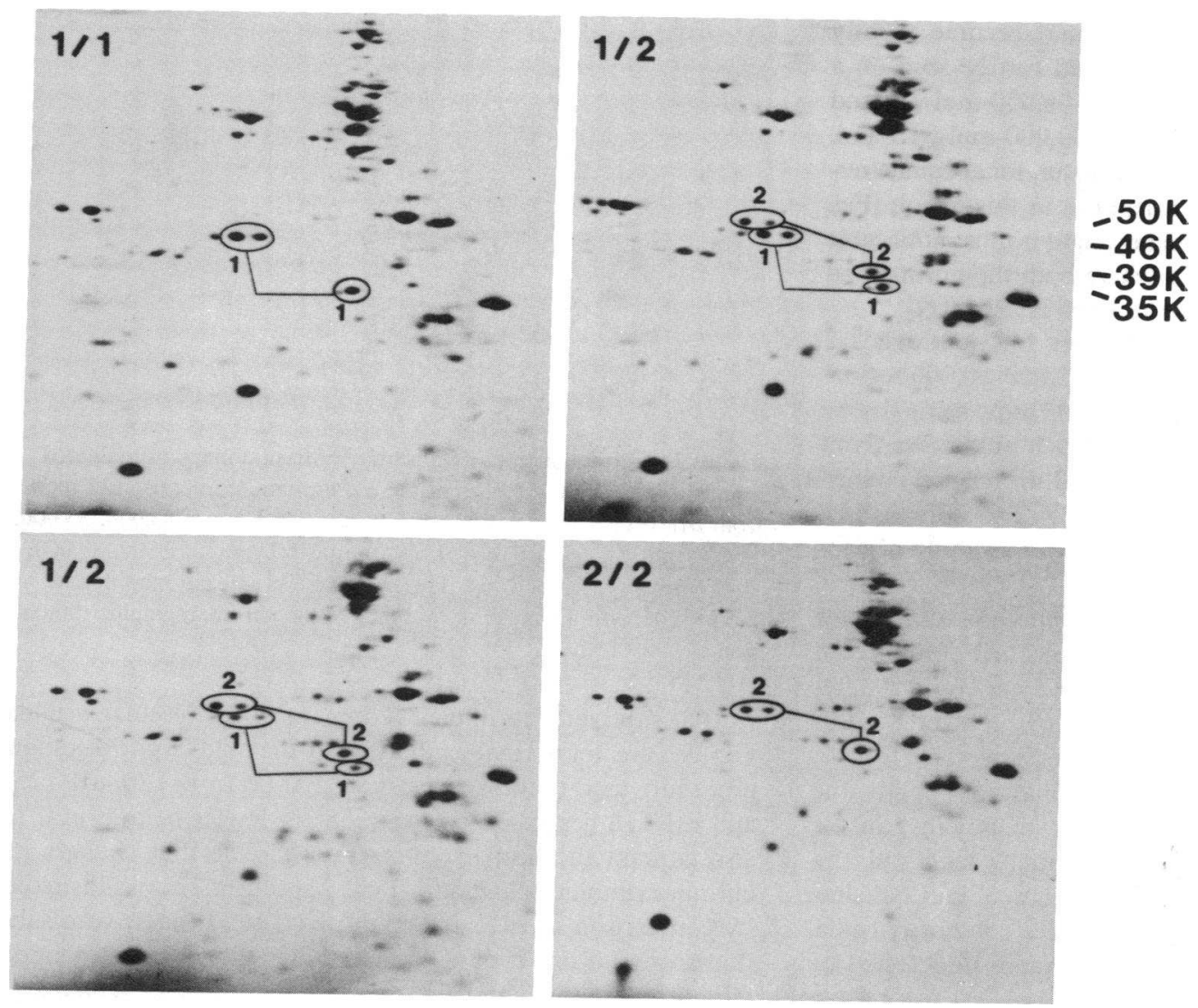

Figure 4. Two-dimensional IEF/ SDS-PAGE showing homozygous and heterozygous Type 2 polymorphism in the $\alpha$ II domain. The parent domains ( $\alpha \mathrm{II}-$ T46 and $\alpha$ II-T50, Type 2), are circled and connected by lines to the subdomains $\alpha$ II-T35 and $\alpha$ II-T39 (Type 2), respectively. Gel conditions are as in Fig. 1 $B ; \alpha$ II types are designated as in Fig. 3. 
and it is this technique that we have used to analyze the structure of the spectrin molecule. In earlier work in our laboratory that combined two-dimensional electrophoresis and peptide mapping, we identified the origin and relationships of the majority of tryptic peptides separated on two-dimensional gels (8-10). The unique $\alpha$ - and $\beta$-chain domains are identified in Fig. $1 B$ and in diagrammatic form in Fig. 2.

In analyzing spectrin from normal volunteers and from families with HS, HE, and HPP, we have detected a polymorphism within the $\alpha$ II domain. The common $\alpha$ II tryptic domain of spectrin from 60 Caucasian donors has a molecular weight of $46,000(\alpha \mathrm{II}-\mathrm{T} 46)$ and is called Type 1 (Fig. $1 B)$. This peptide has two $\mathrm{p} \Gamma \mathrm{s}$, possibly owing to variable deamidation, which are identical by peptide mapping (data not shown). Tryptic digestion at $0^{\circ} \mathrm{C}$ produces a subfragment of $\alpha \mathrm{II}$ that has a molecular weight of 35,000 ; peptides with molecular weights of 30,000 , 25,000 , and 16,000 can be produced by more extensive tryptic digestion. Peptide maps (Fig. 5) have confirmed that the lower molecular weight peptides are fragments of the 46,000-mol-wt domain, and a monoclonal antibody has been made which reacts with a determinant common to all but the 16,000 -mol-wt fragment (10).

The $\alpha$ II domain of spectrin from 29 of 37 black donors studied shows alterations in molecular weight and/or $\mathrm{p} I$. These variations occur in what appear to be heterozygous and homozygous forms. In the most common form of the variant, Type 2, the $\alpha$ II-T46 domain and the $\alpha$ II-T35 subdomain increase in apparent molecular weight by 4,000 and become slightly more basic. The $\alpha$ II Type 2 polymorphism can be seen on a one-dimensional gel (Fig. 3) by the loss of a 46,000-mol-wt band in homozygotes and the presence of a 39,000-mol-wt band. Two-dimensional maps are required, however, for adequate resolution and analysis of individual peptides in this region (Fig. 4). The difference in $\mathrm{p} I$ and molecular weight is most noticeable in the heterozygous condition $1 / 2$, where both the normal and variant peptides are found (Fig. 4, sections labeled 1/2).

Other differences among the $\alpha$ II Types $1 / 1,1 / 2$, and $2 / 2$ appear on the one-dimensional gels in the 70,000-90,000 region. Two-dimensional gels from normal controls show some degree of variability in this group of peptides, which are known from limit peptide mapping to represent several different domains. Using one monoclonal antibody to one $\alpha$ II-T46 domain (see reference 10 ), we detected no $\alpha$ II peptides in this group of peptides.

Limit peptide maps of variant $\alpha$ II tryptic domains. Limit chymotryptic maps of the Type 1 and Type $2 \alpha$ II domains are shown in Fig. 5. Maps of the 46,000-mol-wt (Type 1) and 50,000mol-wt (Type 2) peptides (Fig. 5, $A$ and $B$ ), and of 35,000mol-wt (Type 1) and 39,000-mol-wt (Type 2) peptides (Fig. 5, $C$ and $D$ ) show a difference in a single iodinatable peptide, the Type 2 variant having a relative decrease in mobility in the chromatographic dimension. Since these maps show only iodinatable peptides, predominantly those that contain tyrosine, and on the average only $33 \%$ of all peptides contain tyrosine, other peptides may also be altered but remain undetected by this procedure.



Figure 5. Autoradiographs of limit chymotryptic maps of iodinated intermediate tryptic peptides of the $\alpha$ II domain: comparison of maps from $\alpha$ II Type 1 and Type 2. $A, C, E$ are $\alpha$ II Type 1: 46,000, 35,000, $16,000 \mathrm{~mol} \mathrm{wt}$, respectively. $B, D, F$ are $\alpha$ II Type $2: 50,000,39,000$, $20,000 \mathrm{~mol} w \mathrm{wt}$, respectively. The small arrows in each map denote the peptide with a change in mobility in the chromatographic dimension.

The subfragments of the $\alpha$ II domain with molecular weights $<35,000$ are not normally produced by the mild tryptic digestion procedure used in these experiments. Type $1 \alpha \mathrm{II}-\mathrm{T} 30, \alpha \mathrm{II}-\mathrm{T} 25$, and $\alpha \mathrm{II}-\mathrm{T} 16$ generated by digestion for longer periods at $0^{\circ} \mathrm{C}$ also contain the limit chymotryptic peptide that changes its chromatographic behavior in Type 2 spectrin. This is illustrated by comparison of $\alpha$ II subdomain $\alpha$ II-T16, Type 1, with subdomain $\alpha$ II-T20, Type 2 , which shows the expected peptide variation (Fig. 5, $E$ and $F$ ). 
Variant $\alpha I I$ domains are also produced by $\mathrm{CNBr}$ cleavage. Since the $\alpha$ II peptides discussed above were generated by tryptic digestion, it seemed possible that a lysine or arginine normally present on one end of the $\alpha$ II domain was either buried or substituted in the Type 2 variant. Tryptic digestion would then have cleaved the next lysine or arginine, possibly producing a slightly larger $(50,000$-mol-wt) domain. To characterize further the variant $\alpha$ II domains, spectrin homozygous for Type 1 or Type 2 was cleaved with $\mathrm{CNBr}$ in $70 \%$ formic acid. All peptides that resulted from the cleavage were identical with one exception: Type 1 spectrin contained a 23,000-mol-wt peptide that was slightly more acidic than a corresponding 26,000 -mol-wt peptide in Type 2 spectrin. Limit chymotryptic peptide maps of these $\mathrm{CNBr}$ peptides showed that both 23,000-mol-wt and 26,000mol-wt peptides are derived from the $\alpha$ II domain and that they differ in the mobility of one chymotryptic peptide in the same manner as the maps of the 46,000-mol-wt (Type 1) and 50,000mol-wt (Type 2) tryptic peptides (Fig. 6, $A$ and $B$ ). The preliminary interpretation of these findings is that the $\alpha$ II variants are produced not simply by burial or substitution of a single lysine or arginine, but that the polypeptide chain in this region is increased in apparent molecular weight by approximately 4,000 .

Family studies of spectrin $\alpha I I$ variants. The initial family in which we detected the $\alpha$ II Type 2 variation was a black family with classical HS (Fig. 7). A father and three of the five children tested had classical HS: the three affected brothers had spherocytes on smear, abnormal osmotic fragility tests, and hematocrits of 34-36\% with elevated reticulocyte counts. Two of the sons and the father had had their spleens removed. The two unaffected daughters had normal osmotic fragility tests and one had a hematocrit of $40 \%$ in 1976 at age 23 . When studied in 1981, both daughters had mild anemia with hematocrits 36$37 \%$. Red cell indices, peripheral smears, ferritin, and transferrin levels suggested mild iron deficiency.

When we examined the tryptic digest of spectrin from this family by two-dimensional IEF/SDS-PAGE, we noted $\alpha$ II-T46 Type 2 in either the heterozygous or homozygous form in five of seven family members (Figs. 4 and 7). The distribution of $\alpha$ II-T46 Type 2 followed classical Mendelian genetics but did not correlate with the presence of spherocytosis. The two children who were homozygous for the $\alpha$ II-T46 variant did not have HS.

We subsequently demonstrated other structural variations within the $\alpha$ II domain of black donors. In the Type 3 variation, only the molecular weight of the domain changes (increasing by 4,000 ), and the $\mathrm{p} I$ remains the same. Type 4 consists of a pI change only, the domain becoming slightly more basic. Fig. 8 shows the spectrin maps of donors whose spectrin has both $\alpha$ II Types 1 and $3(1 / 3)$, Types 3 and $4(3 / 4)$, and Types 2 and $4(2 / 4)$. The bottom right panel of Fig. 8 is a composite drawing of the relative positions of peptides in $\alpha$ II variants 1 through 4.

Note that the two types of $\alpha$ II peptides found in one individual are not always present in equal quantities (e.g., Fig. 8, $1 / 3$ ), which one would expect if the donor were a true heterozygote. This may be due to different susceptibilities of each variant to tryptic digestion or may suggest that there are more than two alleles for each spectrin subunit in the genome. Athough family studies were incomplete, the $\alpha$ II variants observed were consistent with simple Mendelian genetics in six families studied.

The distribution of variant spectrins seen in the 37 black individuals (14 kindreds) studied is indicated below:

$\begin{array}{llll}\text { Type-No. } & \text { Type-No. } & \text { Type-No. } & \text { Type-No. } \\ 1 / 1-8 & 2 / 2-3 & 3 / 3-0 & 4 / 4-0 \\ 1 / 2-17 & 2 / 3-3 & 3 / 4-1 & \\ 1 / 3-1 & 2 / 4-1 & & \\ 1 / 4-3 & & & \end{array}$

The polymorphic spectrins were discovered during a study of patients with HS, HE, and related disorders; three of the black families studied have HS, one has HE, and three have HE and HPP, representing 27 of the 37 black individuals. The presence of the $\alpha$ II variant did not appear to affect the clinical presentation of these disorders.


Figure 6. Autoradiographs of limit chymotryptic maps of iodinated $\mathrm{CNBr}$ cleavage products. (A) $\alpha$ II Type 1, 23,000-mol-wt peptide. (B) $\alpha$ II Type 2, 26,000-mol-wt peptide. Arrows denote peptide with change in mobility in the chromatographic dimension. 


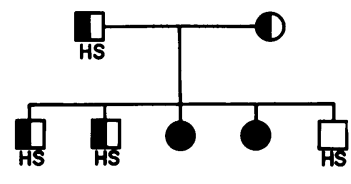

$\alpha$ II Type 1
2
$1 / 2$

Figure 7. Genealogy of family with classical HS and $\alpha$ II Type 2 polymorphism.

\section{Discussion}

The search for structural abnormalities in a large molecule such as spectrin requires a method that not only surveys the entire molecule but that is sensitive enough to pick up small changes in molecular weights or $\mathrm{p} I$ within a domain. The analysis of intermediate tryptic peptides of native spectrin by two-dimensional electrophoresis described above appears to be one method suitable for this task. Furthermore, it is compatible with small volumes of blood obtainable from patients (including children) with hemolytic anemias. The peptides are well resolved and the maps are highly reproducible.

In normal spectrin, most of the lysine and arginine residues are protected from tryptic digestion by the native conformation of the molecule. Small changes in sequence, abnormal subunit association, or oxidative damage could change conformation enough to expose or bury an arginine or lysine residue and thereby result in altered tryptic digestion of native spectrin. For example, in spectrin obtained from patients with HPP, an abnormal conformation within the $\alpha \mathrm{I}-\mathrm{T} 80$ domain causes a new tryptic cleavage which generates two peptides not obtained from normal spectrin (2).

The $\alpha$ II variants illustrated above demonstrate well the sensitivity of this method to small structural changes in spectrin. In contrast to the findings in HPP spectrin, however (2), the $\alpha$ II variants are not the result of new tryptic cleavages. The $\alpha$ II polymorphisms described here consist of two distinct structural changes, a molecular weight increase, as in Type 3, and a basic shift in pI, as in Type 4. The Type $2 \alpha$ II variant, which has both the molecular weight and $\mathrm{p} I$ change, appears to be the result of two changes within a single domain. The limit peptide maps and the cyanogen bromide digests from $\alpha$ II Type 2 spectrin suggest that the alterations are not the result of burial or substitution of a lysine or arginine residue, but are due, at least in part, to a sequence alteration that increases its apparent molecular weight by 4,000 . This apparent increase in molecular weight may be the result of a 4,000-mol-wt insert (roughly 35 amino acids) possibly occurring at an exon-intron interface or at an mRNA splice junction. Alternatively, specific amino acid substitutions have been shown to increase the apparent molecular weight of a protein by 2,000 (11). The change in $\mathrm{p} I$ may occur at a different site within the polypeptide chain, but it is present in the first major cleavage product ( $\alpha$ II T35).



Figure 8. Two-dimensional IEF/SDS-PAGE of spectrin from individuals doubly heterozygous for $\alpha$ II variants $1,2,3$, and 4 . A schematic representation of the relative positions of Types $1,2,3$, and 4 peptides is shown in the lower right panel; solid areas are for reference to other spectrin peptides seen on two-dimensional electrophoresis. 
$\alpha$ II Type 2 spectrin occurred most frequently in the black population we studied, the sample size was small, and two kindreds that accounted for 14 of the 37 black individuals studied were predominantly $1 / 2$ variants; therefore, no conclusions can be drawn from this study about frequency of spectrin $\alpha$ II variation in the black population, nor about the most common type of variation.

We observed no change in behavior of the red cell caused by $\alpha$ II polymorphism, at least by clinical criteria. This is in contrast to alterations in the $\alpha \mathrm{I}-\mathrm{T} 80$ domain that are associated with HPP and HE (2, 3 and Knowles, W. J., J. S. Morrow, S. L. Marchesi, and V. T. Marchesi, manuscript in preparation). Although many of our HS, HE, and HPP patients have polymorphic $\alpha$ II domains, we do not yet have enough data to determine whether this subtly influences the occurrence or expression of these hereditary hemolytic anemias. The question arises as to whether polymorphism such as that seen in the $\alpha$ II domain and a structural change causing hemolytic anemia must occur on the same polypeptide chain for amplification of the potential for hemolysis. Alternatively, does a polymorphism on one subunit increase the hemolytic potential of a structural change on the other subunit? These questions will be answered by continuing phenotypic and genotypic analysis of spectrin and other structural proteins of the red cell membrane.

\section{Acknowledgments}

The authors would like to acknowledge Dr. David Speicher for his help in analyzing the peptide maps. We also acknowledge the assistance of Drs. Joseph Bove, David Seligson, and Peter McPhedran in collecting blood samples and in performing clinical laboratory tests.

This work was funded by U. S. Public Health Service grant AM27932.

\section{References}

1. Marchesi, V. T. 1983. The red cell membrane skeleton: recent progress. Blood. 61:1-11.

2. Knowles, W. J., J. S. Morrow, D. W. Speicher, H. S. Zarkowsky, N. Mohandas, W. C. Mentzer, S. B. Shohet, and V. T. Marchesi. 1983. Molecular and functional changes in spectrin from patients with hereditary pyropoikilocytosis. J. Clin. Invest. 71:1867-1877.

3. Lawler, J., S.-C. Liu, J. Palek, and J. Prchal. 1982. Molecular defect of spectrin in hereditary pyropoikilocytosis: alterations in the trypsin-resistant domain involved in spectrin self-association. J. Clin. Invest. 70:1019-1030.

4. Litman, D., C. J. Hsu, and V. T. Marchesi. 1980. Evidence that spectrin binds to macromolecular complexes on the inner surface of the red cell membrane. J. Cell Sci. 42:1-21.

5. Laemmli, U. K. 1970. Cleavage of structural proteins during the assembly of the head of bacteriophage T4. Nature (Lond.). 227:680685 .

6. O'Farrell, P. 1975. High resolution two-dimensional electrophoresis of proteins. J. Biol. Chem. 250:4007-4021.

7. Elder, J. H., R. A. Pickett, J. Hampton, and R. A. Lerner. 1977. Radio-iodination of protein in single polyacrylamide gel slices. J. Biol. Chem. 252:6510-6516.

8. Speicher, D. W., J. S. Morrow, W. J. Knowles, and V. T. Marchesi. 1980. Identification of proteolytically resistant domains of human erythrocyte spectrin. Proc. Natl. Acad. Sci. USA. 77:5673-5677.

9. Speicher, D. W., J. S. Morrow, W. J. Knowles, and V. T. Marchesi. 1982. A structural model of human erythrocyte spectrin: alignment of chemical and functional domains. J. Biol. Chem. 257:9093-9101.

10. Yurchenco, P. D., D. W. Speicher, J. S. Morrow, W. J. Knowles, and V. T. Marchesi. 1982. Monoclonal antibodies as probes of domain structure of the spectrin subunit. J. Biol. Chem. 257:9102-9107.

11. Noel, D., K. Nikaido, and G. Ames. 1979. A single amino acid substitution in a histidine-transport protein drastically alters its mobility in sodium dodecyl sulfate-polyacrylamide gel electrophoresis. Biochemistry. 18:4159-4165. 